\title{
Shaping the Ultrafast Temporal Correlations of Thermal-Like Photons
}

\author{
Víctor Torres-Company, ${ }^{1, *}$ Juan P. Torres, ${ }^{2,3}$ and Ari T. Friberg ${ }^{4,5, \dagger}$ \\ ${ }^{1}$ School of Electrical and Computer Engineering, Purdue University, West Lafayette, Indiana 47907, USA \\ ${ }^{2}$ ICFO-Institut de Ciencies Fotoniques, Castelldefells, 08860 Barcelona, Spain \\ ${ }^{3}$ Department of Signal Theory and Communications, Universitat Politecnica Catalunya, Campus Nord D3, 08034 Barcelona, Spain \\ ${ }^{4}$ Department of Applied Physics, Aalto University, Box 13500, FI-00076 Aalto, Finland \\ ${ }^{5}$ Department of Physics and Mathematics, University of Eastern Finland, Box 111, FI-80101 Joensuu, Finland
}

(Received 2 May 2012; published 14 December 2012)

\begin{abstract}
We show that the temporal correlations between two light beams arising from a broadband thermal-like source can be controlled in the femtosecond regime. Specifically, by introducing spectral phase-only masks in the path of one of the beams, we show that the timing and strength of the photon correlations can be programmed on demand. This example demonstrates that the interbeam second-order coherence function propagates as a phase-sensitive ultrafast wave packet in the path towards the detectors, and is thus, susceptible to be modified by acting on just one of the beams. For quite some time, it has been thought that this could only happen with sources showing time-energy entanglement. Our work shows that such a property is due to the existence of a certain type of correlation, but not necessarily the entanglement.
\end{abstract}

DOI: 10.1103/PhysRevLett.109.243905

PACS numbers: $42.25 . \mathrm{Kb}, 42.25 . \mathrm{Hz}, 42.50 . \mathrm{Ar}$

Introduction.-The statistical properties of broadband light sources play a key role in understanding the physics behind their emission mechanisms. The theory of optical coherence provides a convenient mathematical framework to account for these stochastic effects by means of a hierarchy of correlation (or coherence) functions [1,2]. The first-order coherence function quantifies the electric field correlations of the source and is used to describe the field superposition effects that appear in the most common interferometers. In the temporal domain, the interference effects of broadband light sources can easily be controlled on demand, thanks to the availability of programmable pulse shapers [3]. Conversely, the measurement of the first-order correlation function yields invaluable information about the interaction of light with matter, and it lies at the heart of some of the most popular bioimaging tools such as optical coherence tomography [4] or spectral endoscopy [5], just to name a few.

The second-order correlation function [1,2] provides a unique fingerprint of the fundamental properties of any radiation source. For example, for thermal-like light sources, this quantity explains why it is twice more probable to detect two photons in coincidence when single-photon detectors are located equidistantly from the source than in any other situation. This effect was first observed in the spatial domain by the groundbreaking experiments of Hanbury Brown and Twiss (HBT) [6]. They showed that the coherence area was related to the angular size of the radiation source [7]. Interestingly, a similar effect also appears in the temporal domain [8], i.e., for thermal-like sources, the photons appear correlated for time instants shorter than the inverse of the bandwidth of the source. On the contrary, for fermions [9] and photons showing sub-Poisson statistics [10], the effect is just the opposite.

The study of higher-order correlations of different types of light sources has been an active area of research for more than six decades and it continues attracting attention due to its relevance in fundamental science $[11,12]$. However, the measurement of second- and higher-order coherence functions of broadband light sources is especially challenging since, due to their large bandwidths, ultrafast (typically in the femtosecond regime) detection schemes are needed [13]. In fact, it was not until very recently that the second-order coherence properties of true thermal photons were experimentally observed [8].

In this Letter, we demonstrate the control, at ultrafast scales, of the second-order coherence properties of thermal-like photons. The key enabling point is that the intensity correlations of two incoherent light beams originating in the same thermal source are sensitive to the spectral phase difference between the two beams, an effect pointed out in Ref. [14]. Therefore, the temporal correlations of the photons can be tailored on demand by placing a programmable spectral phase-only filter in the path of just one of the beams. This effect is quite intriguing, since unlike with spectral amplitude masks [15], spectral-phase filters do not alter the hierarchy of correlation functions of the individual light beam on which they act [16].

Theory.-The theory behind this experiment can be readily understood in the framework of statistical optics as the propagation of the correlation of the source's intensity fluctuations [17]. Alternatively, a full quantum formalism might be used instead [18]. Let us consider the scheme of Fig. 1(a). An incoherent broadband light source with 


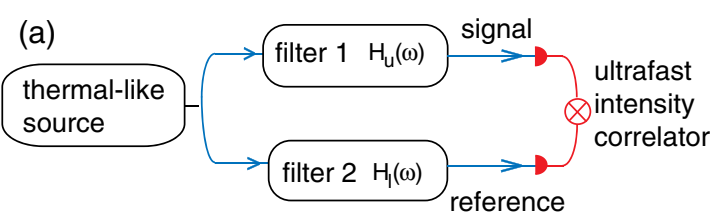

(b)

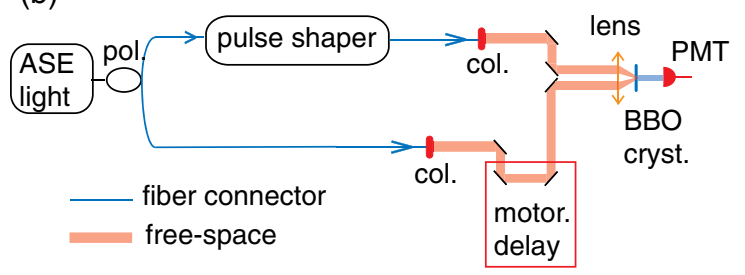

(c)

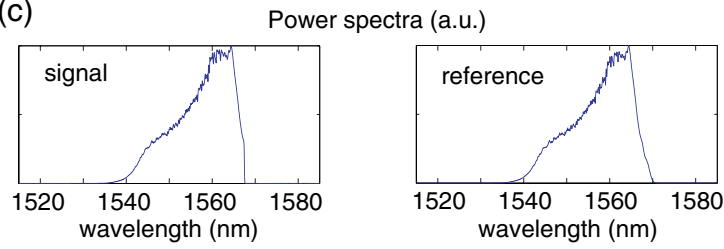

FIG. 1 (color online). (a) Experimental setup to modify and measure the second-order coherence properties of two beams that, while originating in the same thermal-like source, propagate through different paths. Each beam travels through a different medium which can be described mathematically by a complex transfer function $H(\omega)$. Afterwards, the intensity correlations between the two beams are measured with an ultrafast detector, to yield the second-order correlation function $G^{(2)}(\tau)$. (b) Experimental setup. Filter 1 in the signal arm is a programmable pulse shaper that can modify the amplitude and phase of the input broadband spectrum. In the lower arm, a fiber connector is used. The ultrafast intensity cross correlation is performed in the optical domain with a background-free intensity cross correlator with a controllable motorized delay with 1 fs steps. The setup uses an achromatic lens to focus the reference and signal beams onto a $0.5 \mathrm{~mm}$ thick beta-barium borate (BBO) crystal to produce second-harmonic radiation, which is subsequently detected by a photomultiplier tube (PMT). The short length of the beta-barium borate crystal provides broadband phase-matching and femtosecond timing resolution. Further, pol: polarizer; col: fiber collimator. (c) Power spectra (displayed in linear scale) measured at signal and reference arms, respectively.

power spectrum $S(\omega)$ and obeying Gaussian statistics is split into two arms. The effect of propagation in individual arms can mathematically be accounted for by a linear time-invariant filter, with complex optical transfer function $H_{i}(\omega)$, where $i=u, l$ refer to the upper or lower paths. Upon propagation, both light beams are jointly detected by an ultrafast detector and correlated in intensity with each other, leading to a quantity proportional to the secondorder coherence function

$$
G^{(2)}\left(t_{1}, t_{2}\right)=\left\langle I_{u}\left(t_{1}\right) I_{l}\left(t_{2}\right)\right\rangle .
$$

Here, the symbol \langle\rangle denotes ensemble averaging and $I_{u(l)}(t)$ is the optical intensity at the upper (lower) arm. It can be calculated as $I_{u(l)}(t)=\left|E_{u(l)}(t)\right|^{2}$, where

$$
E_{u(l)}(t)=\int d \omega \tilde{E}_{0}(\omega) H_{u(l)}(\omega) \exp (-i \omega t) .
$$

This equation accounts for the propagation of the different realizations of the instantaneous electric field of the source, $E_{0}(t)$, with $\tilde{E}_{0}(\omega)$ denoting the Fourier transform of $E_{0}(t)$. The detected intensity, $I_{u(l)}(t)$, is a random function with ultrafast temporal variations. As is well known, these fluctuations typically occur on the scale on the order of the inverse of the bandwidth of the source [19]. The second-order correlation function can be calculated by substituting Eq. (2) into Eq. (1), leading to

$$
G^{(2)}\left(\tau=t_{2}-t_{1}\right)=I_{u} I_{l}+|\Delta(\tau)|^{2} .
$$

Here,

$$
I_{u(l)}=\int d \omega S(\omega)\left|H_{u(l)}(\omega)\right|^{2}
$$

is the energy carried in the upper (lower) arm and

$$
\Delta(\tau)=\int d \omega S(\omega) H_{u}^{*}(\omega) H_{l}(\omega) \exp (-i \omega \tau)
$$

is the term accounting for the correlation of the intensity fluctuations. To achieve the above results, we have made explicit use of the assumptions that (i) the light source is statistically stationary and (ii) it obeys Gaussian statistics. Condition (i) implies that the source is spectrally uncorrelated, $\left\langle\tilde{E}_{0}^{*}(\omega) \tilde{E}_{0}\left(\omega^{\prime}\right)\right\rangle=S(\omega) \delta\left(\omega-\omega^{\prime}\right)$, while condition (ii) yields by the Gaussian moment theorem that $\left\langle E_{0}^{*}\left(t_{1}\right) E_{0}^{*}\left(t_{2}\right) E_{0}\left(t_{3}\right) E_{0}\left(t_{4}\right)\right\rangle=\left\langle E_{0}^{*}\left(t_{1}\right) E_{0}\left(t_{3}\right)\right\rangle\left\langle E_{0}^{*}\left(t_{2}\right) E_{0}\left(t_{4}\right)\right\rangle+$ $\left\langle E_{0}^{*}\left(t_{1}\right) E_{0}\left(t_{4}\right)\right\rangle\left\langle E_{0}^{*}\left(t_{2}\right) E_{0}\left(t_{3}\right)\right\rangle[1]$.

It is important to note that when no filters are applied, $H_{u(l)}(\omega)=1$, Eqs. (3)-(5) reduce to the well-known result for thermal-like light beams

$$
G^{(2)}(\tau)=I_{0}^{2}+|\Gamma(\tau)|^{2},
$$

with $I_{0}=\Gamma(\tau=0)$ and $\Gamma(\tau)=\int d \omega S(\omega) \exp (-i \omega \tau)$ being the mutual coherence function of the optical source. As one can observe, at $\tau=0$, the function $G^{(2)}(0)=2 I_{0}^{2}$, whereas at time separations $\tau$ much longer than the coherence time of the source, $\tau \gg \tau_{c}$, we have $\Gamma(\tau) \approx 0$ and $G^{(2)}\left(\tau \gg \tau_{c}\right) \approx I_{0}^{2}$. The factor of 2 in the ratio of the correlation function to the background, $g^{(2)}(0)=$ $G^{(2)}(0) / G^{(2)}\left(\tau \gg \tau_{c}\right)$, clearly observed in the experiments of Boitier et al. [12], can thus, be accounted for within a statistical optics framework [13].

Obviously, by placing a narrow band filter, the coherence time of the source is increased and thus, likewise is the temporal window in which the photons appear to be correlated [13,19]. However, Eq. (5) indicates that the second-order coherence function has a more intricate dependence with the spectral phase of the filters placed in the beam paths. This in radical contrast to Eq. (4), which indicates that the mean value of the ultrafast intensity in the individual beams actually does not depend on the spectral 
phase. In fact, the hierarchy of correlation functions of a thermal-like beam remains unaffected by a spectral phaseonly filter [16]. Equations (3)-(5) provide a more general picture of the interbeam temporal correlations, by considering nonequal paths in the propagation towards the detectors, revealing a more subtle spectral phase dependence.

Experiment.-The experimental setup is depicted in Fig. 1(b). The optical source is the amplified spontaneous emission radiation of an erbium-doped fiber amplifier. It has $\sim 200 \mathrm{~mW}$ average power and $2.15 \mathrm{THz}$ bandwidth (measured at full width at half maximum). The thermallike behavior of this kind of source has been demonstrated in different experiments $[12,20]$. The light is polarized and split into two different paths, similarly to the HBT setup of Ref. [6] (Fig. 2).

In one of the arms, a commercially available pulse shaper (Finisar Waveshaper 1000S) is located. This device can program $H_{u}(\omega)$ in both amplitude and phase with $10 \mathrm{GHz}$ resolution within the whole communications $C$ band (1527.4-1567.4 $\mathrm{nm}$ ). We carefully measured the time of flight through the upper arm and then prepared a fiber link of $7.238 \mathrm{~m}$ for the lower arm so that the timing difference between the two arms was located within the range of the motorized stage of the cross correlator. The optical power spectra at different arms are shown in Fig. 1(c). As can be seen, $H_{u}(\omega)$ introduces a slight cutoff at longer wavelengths. This effect can easily be taken into account through Eqs. (2)-(5) and is included in the subsequent simulations. Afterwards, the light beams coming from both arms are sent to a homemade background-free noncollinear intensity cross correlator. This machine has the capability to measure the intensity cross correlation [Eq. (3)] with femtosecond accuracy [21].

The fiber link in the lower arm is placed to adjust the timing. However, it introduces residual dispersion,

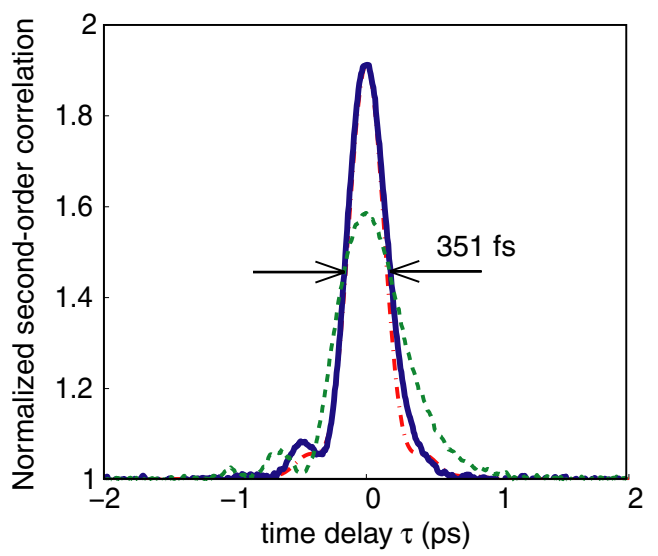

FIG. 2 (color online). Measured normalized second-order correlation function $g^{(2)}(\tau)$ when the spectral phase is corrected with the pulse shaper (continuous blue line), compared with the expected $g^{(2)}(\tau)$ trace (dash-dotted red line). When the programmed phase is reversed in sign, the value $g^{(2)}(0)$ drops and the cross correlation broadens (dotted green). i.e., $H_{l}(\omega)=\exp \left(i \Phi_{2 \text { res }} \omega^{2} / 2\right)$, where the angular frequencies are referred to baseband for simplicity. This problem can be circumvented since, according to Eq. (5), this residual dispersion can be compensated by programming the same dispersion [14] with the pulse shaper $\left(\sim-0.0215 \mathrm{ps}^{2}\right.$, in this case). The resulting compressed cross correlation is displayed in Fig. 2, normalized with respect to the background (solid blue line). It shows a peak with a width of $351 \mathrm{fs}$ and $g^{(2)}(0)=1.91$, very close to the ideal value of 2 . To make sure that we have achieved the shortest possible cross correlation, we compare the result with the expected one taking into account Eqs. (3)-(5) (dash-dotted red line). There is an excellent agreement between the two curves, indicating that the second-order coherence function is indeed "transform-limited". To illustrate the phase-sensitive nature of the cross correlation, we simply reverse the sign of the programmed dispersion with the shaper. The ensuing cross correlation width increases to $494 \mathrm{fs}$ and the peak drops significantly (dotted green curve). In the following, we will add the above required dispersion amount to the different spectral phases implemented with the pulse shaper.

The results shown in Fig. 2 demonstrate that the temporal correlations of two light beams generated by the same thermal source, and thus lacking entanglement, can be tailored via remote control of the dispersion that sees one of the light beams, similarly to the effect observed with entangled two-photon states, such as the ones produced by means of spontaneous parametric down conversion [22,23]. We should remark that this does not mean that the light beams considered here share the same characteristics with the entangled two-photon states. However, both light sources have the required correlations that enable its observation.

In a further step, we illustrate that the photon temporal correlations can be controlled on demand by spectral phase shaping. Concretely, we implement the following spectral phase $H_{u}(\omega)=\exp [i \eta \sin (\omega T)]$, with $\eta$ and $T$ real independent parameters to be programmed with the pulse shaper. Let us show first what we should expect, by introducing this expression into Eq. (5). Since the residual dispersions are compensated we can safely consider $H_{l}(\omega)=1$, thus we get

$$
G^{(2)}(\tau)=I_{0}^{2}+\left|\Delta^{\prime}(\tau)\right|^{2},
$$

with

$$
\Delta^{\prime}(\tau)=\Gamma(\tau) \otimes \sum_{n} J_{n}(\eta) \delta(\tau-n T)
$$

Here, $\otimes$ denotes the convolution operation and $J_{n}$ are the Bessel functions of the first kind. The above equation indicates that, due to the effect of the spectral filter, there appear several temporal locations at which the normalized second-order coherence function shows a peak. These locations are determined through the parameter $T$, which in turn can be programmed by the pulse shaper. Furthermore, the 

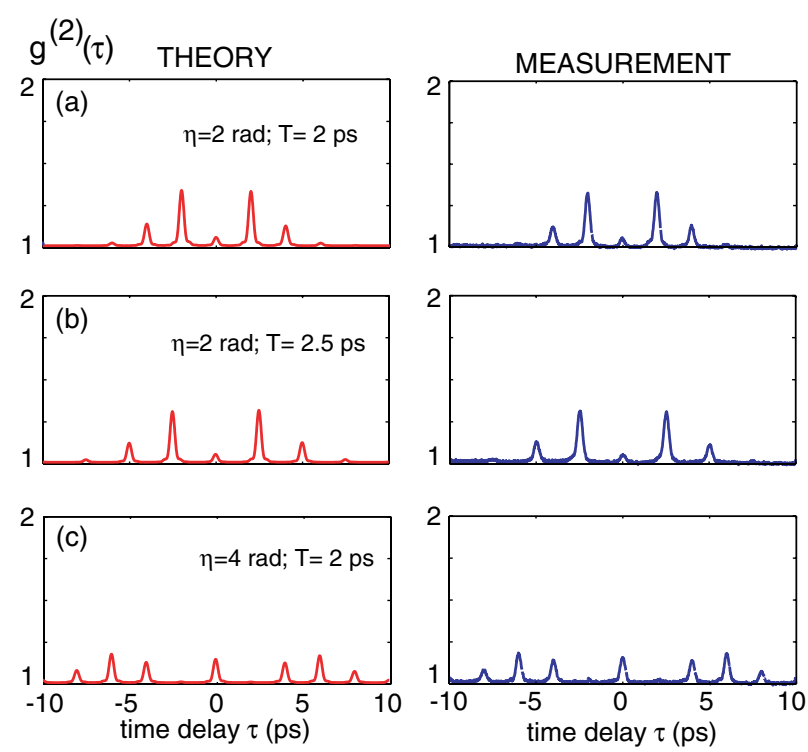

FIG. 3 (color online). Shaping of the photon temporal correlations. Simulation (left column) and measurement (right column) of the normalized second-order correlation function $g^{(2)}(\tau)$ when the pulse shaper is programmed with the spectral phase $\exp [i \eta \sin (\omega T)]$. The relevant parameters appear in the figure.

strength of these peaks can be independently controlled by a suitable choice of the strength parameter $\eta$.

The results of this experiment are presented in Fig. 3. The temporal location of the peaks can be changed with femtosecond accuracy because it is programmed as a phase encoded in the spectral domain, as shown in Figs. 3(a) and 3(b). In Fig. 3(c), we display the effect of changing the strength parameter $\eta$. As expected from the theoretical calculation taking into account Eq. (3), the probability of detecting coincident photons at $\tau=0$ increases with respect to the previous case, where $\eta=2 \mathrm{rad}$. The above example shows that the temporal correlations of the two beams that propagate through different paths can be tailored while leaving completely unaltered the hierarchy of the correlations of the individual light beams. In other words, despite acting on one of the beams, the effect only manifests when performing intensity cross correlations.

Summary.-We have shown that the ultrafast photon temporal correlations (second-order coherence function) of two thermal-like light beams which traverse different paths depend on the spectral phase difference of the filters located in their paths. With the aid of a programmable pulse shaper, we have demonstrated that the strength and the time instant at which the second-order coherence function shows a peak can be controlled on demand in a subpicosecond temporal scale. This physical phenomenon reveals that the second-order coherence properties of two optical beams originating from the same thermal-like source propagate as an ultrashort phase-sensitive wave packet, whose characteristics can be affected while leaving unaltered the hierarchy of correlation functions of the individual beams. This is similar to what happens to the joint amplitude distribution of frequency entangled twophoton states propagating in a dispersive medium [24], whose joint correlations can also be described as a wave packet that propagates along the medium.

Discussion.-The possibility of tailoring the temporal correlations of light beams on demand is a necessary ingredient for the exploration and development of quantum-inspired classical technologies, i.e., applications with highly beneficial capabilities that can be understood with the laws of classical physics to emulate effects first revealed in the realm of quantum mechanics [25]. This is the case, for instance, of two-photon lithography [26,27] and remote dispersion and temporal modulation cancellation $[18,22,28,29]$. In all these cases, classical and quantum sources share the same enabling characteristics (certain frequency correlations) that allow us the sought-after observation. Importantly, the classical source generally shows a higher brightness and easier implementation than its quantum counterpart, facilitating the realization of the system.

One paradigmatic example is quantum optical coherence tomography [30], a counterpart quantum version of the usual optical coherence tomography (OCT) technique, which makes use of broadband entangled photon pairs to eliminate the disturbing decrease of sensitivity in OCT that usually accompanies the presence of chromatic dispersion. Notwithstanding, correlations between light beams, and not entanglement, is the key enabling factor behind dispersion compensation in OCT [31]. Therefore, a beneficial effect first put forward in a quantum context, could also be implemented with a more convenient, i.e., with a higher intensity, light source.

V. T.-C. is particularly grateful to Andrew M. Weiner for letting him use the equipment at the Ultrafast Optics Laboratory in Purdue University to perform this experiment. J.P. T. acknowledges support from the Government of Spain (project FIS2010-14831), project FET-Open 255914 (PHORBITECH) from the European Union and Fundacio Privada Cellex Barcelona. A. T. F. acknowledges support from the Academy of Finland.

*Present address: Microtechnology and Nanoscience Department, SE-41296 Gothenburg, Sweden. torresv@chalmers.se

${ }^{\dagger}$ Also with the Department of Microelectronics and Applied Physics, Royal Institute of Technology (KTH), Electrum 229, SE-164 40 Kista, Sweden.

[1] L. Mandel and E. Wolf, Optical Coherence and Quantum Optics (Cambridge University Press, Cambridge, England, 1995).

[2] R. J. Glauber, Phys. Rev. 130, 2529 (1963).

[3] V. Binjrajka, C. C. Chang, D. E. Leaird, and A. M. Weiner, Opt. Lett. 21, 1756 (1996).

[4] A.F. Fercher, W. Drexler, C. K. Hitzenberger, and T. Lasser, Rep. Prog. Phys. 66, 239 (2003). 
[5] G. J. Tearney, M. Shishkov, and B. E. Bouma, Opt. Lett. 27, 412 (2002).

[6] R. Hanbury Brown and R. Q. Twiss, Nature (London) 177, 27 (1956).

[7] R. Hanbury Brown and R. Q. Twiss, Nature (London) 178, 1449 (1956).

[8] F. Boitier, A. Godard, E. Rosencher, and C. Fabre, Nat. Phys. 5, 267 (2009).

[9] M. Henny, S. Oberholzer, C. Strunk, T. Heinzel, K. Ensslin, M. Holland, and C. Schonenberger, Science 284, 296 (1999); H. Kiessel, A. Renz, and F. Hasselbach, Nature (London) 418, 392 (2002); W. D. Oliver, J. Kim, R. C. Liu, and Y. Yamamoto, Science 284, 299 (1999); T. Jeltes, J.M. McNamara, W. Hogervost, W. Vassen, V. Krachmalnicoff, M. Schellekens, A. Perrin, H. Chang, D. Boiron, A. Aspect, and C. I. Westbrook, Nature (London) 445, 402 (2007).

[10] H. J. Kimble, M. Dagenais, and L. Mandel, Phys. Rev. Lett. 39, 691 (1977); S. L. Mielke, G. T. Foster, and L. A. Orozco, Phys. Rev. Lett. 80, 3948 (1998); K. M. Birnbaum, A. Boca, R. Miller, A. D. Boozer, T.E. Northup, and H. J. Kimble, Nature (London) 436, 87 (2005).

[11] Y. Bromberg, Y. Lahini, E. Small, and Y. Silberberg, Nat. Photonics 4, 721 (2010).

[12] F. Boitier, A. Godard, N. Dubreuil, P. Delaye, C. Fabre, and E. Rosencher, Nat. Commun. 2, 425 (2011).

[13] E. M. Purcell, Nature (London) 178, 1449 (1956).

[14] V. Torres-Company, H. Lajunen, and A. T. Friberg, New J. Phys. 11, 063041 (2009).

[15] M. L. Goldberger, H. W. Lewis, and K. M. Watson, Phys. Rev. 142, 25 (1966).
[16] L. J. Wang, B. E. Magill, and L. Mandel, J. Opt. Soc. Am. B 6, 964 (1989).

[17] V. Torres-Company, H. Lajunen, J. Lancis, and A. T. Friberg, Phys. Rev. A 77, 043811 (2008).

[18] V. Torres-Company, A. Valencia, M. Hendrych, and J. P. Torres, Phys. Rev. A 83, 023824 (2011).

[19] B. L. Morgan and L. Mandel, Phys. Rev. Lett. 16, 1012 (1966).

[20] V. Torres-Company, C. R. Fernandez-Pousa, and J. P. Torres, Opt. Lett. 35, 1850 (2010).

[21] A. M. Weiner, Ultrafast Optics (Wiley InterScience, New York, 2009).

[22] S. Y. Baek, Y.W. Cho, and Y.H. Kim, Opt. Express 17, 19241 (2009).

[23] K. A. O'Donnell, Phys. Rev. Lett. 106, 063601 (2011).

[24] A. Valencia, M. V. Chekhova, A. Trifonov, and Y. Shih, Phys. Rev. Lett. 88, 183601 (2002).

[25] R. Prevedel, K. M. Schreiter, J. Lavoie, and K. J. Resch, Phys. Rev. A 84, 051803(R) (2011).

[26] T. B. Pittman, Y.H. Shih, D. V. Strekalov, and A. V. Sergienko, Phys. Rev. A 52, 3429(R) (1995).

[27] A. Valencia, G. Scarcelli, M. D’Angelo, and Y. Shih, Phys. Rev. Lett. 94, 063601 (2005).

[28] J. Brendel, H. Zbinden, and N. Gisin, Opt. Commun. 151, 35 (1998).

[29] S. Sensarn, G. Y. Yin, and S. E. Harris, Phys. Rev. Lett. 103, 163601 (2009).

[30] M. B. Nasr, B.E. A. Saleh, A. V. Sergienko, and M. C. Teich, Phys. Rev. Lett. 91, 083601 (2003).

[31] J. Le Gouet, D. Venkatraman, F. N. C. Wong, and J. H. Shapiro, Opt. Lett. 35, 1001 (2010). 